Out of 632 samples tested, 26 led to an invalid result (4.1\%) due to a reduced signal for the sample control (SC). A weak PCR performance was observed in $3.5 \%$ of all samples tested. Interestingly, PCR failure rate was more prominent among anal specimens (21.1\%).

Conclusion The high prevalence of HPV infections in men shows that routine testing is justified. FLOQSwabs ${ }^{\circledR}$ proved to be the main criterion for a robust HPV analysis allowing sufficient collection of cellular material. Further studies need to be conducted to improve collection and processing of anal samples to increase number of valid anal samples.

\section{P325 HIV STATUS DISCLOSURE AMONG PREGNANT WOMEN AT A DISTRICT HOSPITAL IN GHANA}

${ }^{1,2}{ }^{2}$ Agyarko-Poku*, ${ }^{1} \mathrm{~F}$ Ankobea, ${ }^{2} \mathrm{R}$ Bandoh, ${ }^{2} \mathrm{E}$ Sorvor. ${ }^{1}$ Kwame Nkrumah University of Science and Technology, Kumasi Ashanti, Ghana; '2Suntreso Government Hospital, Kumasi, Ashanti, Ghana

\subsection{6/sextrans-2021-sti.379}

Background HIV disclosure plays a critical role in the prevention, treatment, care and support for HIV-infected persons. In the context of PMTCT, disclosure to sexual partners has been associated with improved adherence to antiretroviral therapy, better infant feeding practices, safer sex and increased male partner testing. The objective of this study was to assess HIV status disclosure among pregnant women and explore factors that affect disclosure.

Methods A cross sectional institutional based survey was conducted at the Suntreso Government Hospital Kumasi from April to May 2020 using a quantitative approach. Data was collected from pregnant women accessing antenatal care at the selected facility using a structured questionnaire and analysed using STATA 18 version.

Results A total of 118 pregnant women were sampled for the study. Majority (27.97\%) of pregnant women were between 21-25 years. More than half (52.54\%) were married. Almost a half $(48.2 \%)$ had at least a secondary school education. HIV status disclosure rate was $37.29 \%$ and the most predominant reason for nondisclosure was fear of loss of economic support (28.81\%). A third (33.05\%) will never disclose their status. $43.22 \%$, believe antiretroviral therapy could prevent mother to child transmission of HIV. Education, parity and reason for nondisclosure were found to be statistically associated with HIV status disclosure.

Conclusion The high nondisclosure rate among seropositive women in this study highlight the need for interventions that will encourage HIV status disclosure among pregnant women.

\section{P327 DISPARITIES AND TRENDS IN SEXUAL PRACTICES AND STI POSITIVITY AMONG WOMEN ACCORDING TO SEXUAL ORIENTATION IN MELBOURNE, AUSTRALIA, 2011-2019}

\footnotetext{
${ }^{1,2}$ J Engel ${ }^{*},{ }^{1,2} \mathrm{C}$ Fairley, ${ }^{1,2} \mathrm{~K}$ Greaves, ${ }^{1,2} \mathrm{~L}$ Vodstrcil, ${ }^{1,2}$ J Ong, ${ }^{1,2} \mathrm{C}$ Bradshaw, ${ }^{1,2} \mathrm{M}$ Chen, ${ }^{1,2} \mathrm{~T}$ Philips, ${ }^{1,2,3} \mathrm{E}$ Chow. 'Melbourne Sexual Health Centre, Alfred Health, Melbourne, Australia; ${ }^{2}$ Central Clinical School, Monash University, Melbourne, Australia; ${ }^{3}$ Centre for Epidemiology and Biostatistics, Melbourne School of Population and Global Health, The University of Melbourne, Melbourne, Australia
}

10.1136/sextrans-2021-sti.380
Objectives Despite rises in sexually transmitted infection (STI) notifications among Australian women in the previous decade, limited STI surveillance data exists for women by sexual orientation. This study aimed to compare differences in sexual practices and STI positivity among women by sexual orientation, and whether these changed over time.

Methods In this retrospective repeated cross-sectional study, women attending the Melbourne Sexual Health Centre for the first time between 2011-2019 were categorised as 'bisexual', 'lesbian' or 'heterosexual' according to sexual practices in the previous 12 months. Demographic information, sexual practices and STI positivity were compared between the three groups and over time.

Results 36,147 women (2,618 bisexual, 534 lesbian and 32,995 heterosexual) were included. Bisexual women reported more sexual partners (median $=6 ; \mathrm{IQR}=4-10$ ), followed by heterosexual (median=3; IQR =2-5) and lesbian (median=2; $\mathrm{IQR}=1-4$ ) women. A higher proportion of bisexual women consistently used condoms with casual male partners compared to heterosexual women $(20.4 \%$ vs $15.9 \%$; $\mathrm{p}<0.001)$. Consistent condom use with casual male partners decreased over time in heterosexual women, $(19.9 \%$ in 2011 to $15.2 \%$ in 2019, $\mathrm{P}$ [trend $]<0.001$ ) but not in bisexual women. Bacterial vaginosis was more common in lesbian women $(14.8 \%)$ than in bisexual (11.8\%) and heterosexual women (7.7\%) $(\mathrm{p}<0.001)$. Chlamydia was more common in heterosexual women $(9.3 \%)$ than in bisexual $(6.6 \%)$ and lesbian women $(1.2 \%)(p<0.001)$. Syphilis was more common in heterosexual women $(1.0 \%)$ than in bisexual $(0.3 \%)$ and lesbian women $(0.0 \%) \quad(p=0.004)$. Over time, chlamydia positivity in lesbian women increased (from $0.0 \%$ to $2.7 \%$, P[trend] $=0.014$ ), and syphilis positivity in bisexual women increased (from $0.0 \%$ to $0.7 \%$, P [trend] $=0.028$ ), but positivity of these STIs did not change in other groups.

Conclusion Sexual practices and STI positivity differed according to the sexual orientation of women. Knowledge of these differences is important to account for future changes in STI trends that may occur in these subpopulations.

\section{P328 HIV STATUS IS NOT ASSOCIATED WITH TREPONEMA PALLIDUM BURDEN IN EARLY SYPHILIS CASES IN CALI, COLOMBIA}

${ }^{1} S$ Silva Peña* ${ }^{1,2}$ J Garcia Luna, ${ }^{1,2}$ L Ramírez, ${ }^{3} \mathrm{C}$ La Vake, ${ }^{3,4}{ }^{3} \mathrm{~K}$ Hawley, ${ }^{1} \mathrm{E}$ Lopez Medina, 3,4,5 Salazar. 'Centro Internacional de Entrenamiento e Investigaciones Médicas (CIDEIM), Cali, Colombia; ${ }^{2}$ Universidad ICESI, Cali, Colombia; ${ }^{3}$ Department of Pediatrics, University of Connecticut School of Medicine, Farmington, USA; ${ }^{4}$ Division of Pediatric Infectious Diseases and Immunology, Connecticut Children's, Hartford, USA; ${ }^{5}$ Department of Immunology, University of Connecticut School of Medicine, Farmington, USA

\subsection{6/sextrans-2021-sti.381}

Background The re-emergence of venereal syphilis cases is a public health concern, particularly in people living with HIV (PLWH). There is conflicting evidence whether or not Treponema pallidum (Tp) burdens are higher in early syphilis (ES) patients with HIV. The aim of this retrospective study was therefore to compare $\mathrm{Tp}$ burdens in blood and tissues of PLWH and people without HIV (PWH).

Methods Patients with ES were consented and enrolled in Cali, Colombia between 2003 and 2020. Age, sex, HIV status and $\mathrm{Tp}$ polA results were extracted from available clinical and laboratory records. Data from PLWH and PWH were compared using Fisher's exact test for qualitative variables and 